\title{
АСИМПТОТИКА РЕШЕНИЙ РАЗРЫВНЫХ СИНГУЛЯРНО ВОЗМУЩЕННЫХ КРАЕВЫХ ЗАДАЧ
}

Asymptotic expansion is constructed for a solution of boundary-value problem for a singularly perturbed system of differential equations whose right-hand sides are discontinuous on certain surface.

Побудовано асимптотичне розвинення розв'язку крайової задачі для сингулярно збуреної системи диференціальних рівнянь з правою частиною, розривною на певній поверхні.

В данной работе метод пограничных функций А. Б. Васильевой [1] распространяется на нелинейные сингулярно возмущенные краевые задачи для систем, правые части которых имеют разрыв на некоторой поверхности. Асимптотическое разложение решения соответствующей задачи Коши получено в [2].

1. Постановка задачи. Пусть движение некоторого объекта на сегменте $I=$ $=[0, T]$ описывается системой дифференциальных уравнений

$$
\mu \frac{d z}{d t}=F(z, y, t), \quad \frac{d y}{d t}=f(z, y, t),
$$

где

$$
\begin{aligned}
& F \equiv\left\{F^{j}(z, y, t) \mid j=\operatorname{sign} \Phi(z, y, t)\right\}, \\
& f \equiv\left\{f^{j}(z, y, t) \mid j=\operatorname{sign} \Phi(z, y, t)\right\},
\end{aligned}
$$

$\mu>0$ - малый параметр; $z$ и $F-M$-мерные вектор-функции; $y$ и $f-m$-мерные вектор-функции; $\Phi(z, y, t)-$ скалярная функция; $\Gamma=$ $=\{(z, y, t) \mid \Phi(z, y, t)=0\}-$ поверхность переключения. Краевые условия имеют вид

$$
\Psi(x(0, \mu), x(T, \mu), \mu)=0 .
$$

Здесь $\Psi-(M+m)$-мерная вектор-функция, $x=(z, y)$. Решение $x(t, \mu)$ краевой задачи (1) - (3) рассматривается в классе абсолютңо непрерывных функций, удовлетворяющих условию (3) и почти всюду на $I$ системе уравнений (1). Предположим, что для любого $\bar{y}_{0}^{0}$ существует решение вырожденной задачи

$$
0=F\left(\bar{z}_{0}(t), \bar{y}_{0}(t), t\right), \quad \frac{d \bar{y}_{0}}{d t}=f\left(\bar{z}_{0}(t), \bar{y}_{0}(t), t\right), \quad \bar{y}_{0}(0)=\bar{y}_{0}^{0},
$$

пересекающее поверхность $\Gamma$ в некоторый момент времени $t_{0}^{1} \in \operatorname{int} I$. Кроме пограничных слоев, возникающих в соответствующих непрерывных задачах, в данной задаче явление пограничного слоя происходит и в окрестности точки $t_{0}^{1}$. Пограничные функции $\Pi_{0} z^{j}\left(\tau_{0}^{j}\right)$, определяющие поведение решения $z(t$, н) задачи (1) - (3) в зоне пограничного слоя, при $\tau_{0}^{j}>0 ; j=0,1$, удовлетворяют задачам

$$
\begin{gathered}
\frac{d \Pi_{0} z^{j}}{d \tau_{0}^{j}}=F\left(\bar{z}_{0}\left(t_{0}^{j}+0\right)+\Pi_{0} z^{j}, \bar{y}_{0}\left(t_{0}^{j}\right), t_{0}^{j}\right), \\
\Pi_{0} z^{j}(0)=\bar{z}_{0}\left(t_{0}^{j}-0\right)-\bar{z}_{0}\left(t_{0}^{j}+0\right),
\end{gathered}
$$

где $t_{0}^{0} \equiv 0, \bar{z}\left(t_{0}^{0}-0\right) \equiv \bar{Z}_{0}^{0}, \quad \tau_{0}^{j}=\left(t-t_{0}^{j}\right) / \mu$. 
Асимптотическое разложение решения $x(t, \mu)$ краевой задачи (1) - (3) ищется как решение задачи (1), (2) с начальным условием

$$
x(0, \mu)=\sum_{l=0}^{\infty} \mu^{l} \bar{X}_{l}^{0},
$$

причем коэффициенты $\bar{X}_{l}^{0}$ определяются из условия (3). Асимптотическое представление решения $x(t, \mu)$ сингулярно возмущенной задачи Коши (1), (2), (6) построено в [2] и имеет вид

$$
x^{j}(t, \mu)=\sum_{l=0}^{\infty} \mu^{l} \bar{x}_{l}^{j}(t)+\sum_{l=0}^{\infty} \mu^{l} \Pi_{l} x^{j}\left(\tau^{l}\right),
$$

где $\bar{x}_{0}(t) \equiv \bar{x}_{0}^{0}(t)$ при $0 \leq t<t_{0}^{1}, \bar{x}_{0}(t) \equiv \bar{x}_{0}^{1}(t)$ при $t_{0}^{1}<t \leq T, x(t, \mu) \equiv x^{0}(t, \mu)$ при $0 \leq t<t_{1}(\mu), x(t, \mu) \equiv x^{1}(t, \mu)$ при $t_{1}(\mu) \leq t \leq T$,

$$
\tau^{j}=\left(t-t^{j}\right) / \mu, \quad t^{0}=t_{0}^{0}=0, \quad\left(x\left(t^{1}, \mu\right), t^{1}\right) \in \Gamma, \quad t^{1}(\mu)=\sum_{l=0}^{\infty} \mu^{l} t_{l}^{1} .
$$

Подставив представление (7) в условие (3) и разложив в ряд по степеням $\mu$, получим

$$
\sum_{l=0}^{\infty} \mu^{l} \Psi_{l}=0
$$

откуда уравнения $\Psi_{l}=0$ определяют коэффициенты $\bar{X}_{l}^{0}$. Заметим, что

$$
\Psi_{0} \equiv \Psi\left(x_{0}, \bar{x}_{0}(T), 0\right)=0 .
$$

2. Теорема об оценке остаточного члена асимптотического разложения. Введем условие [1] разрешимости краевой задачи (1) - (3) при соответствующем выборе коэффициентов $\bar{X}_{l}^{0}$ ряда (6) и условия [2] для асимптотического представления решения $x(t, \mu)$ задачи Коши (1), (2), (6).

1. Система (8) относительно $x_{0}$ имеет решение $x_{0}=\bar{X}_{0}^{0}$ и функциональный определитель

$$
\left.\Lambda_{0}\left(\bar{X}_{0}^{0}\right) \equiv \frac{D\left(\Psi_{0}\right)}{D\left(x_{0}\right)}\right|_{x_{0}=\bar{X}_{0}^{0}} \neq 0 .
$$

2. Функции $F^{ \pm 1}, f^{ \pm 1}, \Phi, \Psi n+2$ раза непрерывно дифференцируемы в некоторой открытой области $Q \subset R^{M+m+1}$, причем

$$
F^{j} \equiv a_{1}^{j}(y, t) z+a_{2}^{j}(y, t), \quad f^{j} \equiv a_{3}^{j}(y, t) z+a_{4}^{j}(y, t) .
$$

3. Уравнения $F^{ \pm 1}(z, y, t)=0$ имеют $n+2$ раза непрерывно дифференцируемые изолированные решения $z=\varphi^{ \pm 1}(y, t)$ на непустом компактном множестве $Q \subset R^{m+1}$ и точка $(\varphi(y, t), y, t) \in Q$, если $(y, t) \in Q_{1}$, где $\varphi \equiv$ $\equiv\left\{\varphi^{j}(y, t) \mid j=\operatorname{sign} \Phi\left(\varphi^{j}(y, t), y, t\right)\right\}$.

4. Вырожденная задача (4) на промежутке $I$ имеет решение $\bar{z}_{0}(t)=$ $=\varphi\left(\bar{y}_{0}(t), t\right), \bar{y}_{0}(t)$ такое, что точка $\left(\bar{y}_{0}(t), t\right) \in Q_{1}$ и существует $t_{0}^{1} \in \operatorname{int} I$, что

$$
\lim _{t \rightarrow t_{0}^{1}-0} \Phi\left(\bar{z}_{0}(t), \bar{y}_{0}(t), t\right)=0, \quad \lim _{t \rightarrow t_{0}^{1}-0} \frac{d \Phi\left(\bar{z}_{0}(t), \bar{y}_{0}(t), t\right)}{d t}=\alpha \neq 0,
$$




$$
\Phi\left(\bar{Z}_{0}^{0}, \bar{Y}_{0}^{0}, 0\right) \cdot \Phi\left(\bar{z}_{0}(0), \bar{Y}_{0}^{0}, 0\right)>0, \quad \Phi\left(\bar{z}_{0}(\xi), \bar{y}_{0}(\xi), \xi\right) \cdot \Phi\left(\bar{z}_{0}(\eta), \bar{y}_{0}(\eta), \eta\right)<0
$$

при $\xi \in\left[0, t_{0}^{1}\right), \eta \in\left(t_{0}^{1}, T\right]$.

5. Собственные значения $\bar{\lambda}_{k}(t)$ матрицы $F_{z}\left(\bar{z}_{0}(t), \bar{y}_{0}(t), t\right)$ при $t \in I$ удовлетворяют неравенствам $\operatorname{Re} \bar{\lambda}_{k}(t)<0, k=\overline{1, M}$.

6. Для решений $\Pi_{0} z^{j}\left(\tau_{0}^{j}\right)$ задач (5) при $j=0,1$ выполняются условия

$$
\begin{gathered}
\left(\bar{z}_{0}\left(t_{0}^{j}+0\right)+\Pi_{0} z^{j}\left(\tau_{0}^{j}\right), \bar{y}_{0}\left(t_{0}^{j}\right), t_{0}^{j}\right) \in Q \text { при } \tau_{0}^{j} \geq 0 ; \\
\lim _{\tau_{0}^{j} \rightarrow \infty} \Pi_{0} z^{j}\left(\tau_{0}^{j}\right)=0 ;
\end{gathered}
$$

существует $\varepsilon_{0}>0$ такое, что при $t \in\left(t_{0}^{1}, t_{0}^{1}+\varepsilon_{0}\right)$

$$
\alpha \lim _{\mu \rightarrow 0} \frac{d}{d t} \Phi\left(\bar{z}_{0}(t)+\Pi_{0} z^{1} \frac{\left(t-t_{0}^{1}\right)}{\mu}, \bar{y}_{0}(t), t\right)>0 .
$$

Теорема. Пусть для некоторого $\bar{X}_{0}^{0}$ выполняются условия $1-6$. Тогда существуют постоянные $C>0, \delta>0, \mu_{0}>0, \alpha>0$ такие, что для $\mu \in$ $\in\left(0, \mu_{0}\right]$ в $\delta$-окрестности множества

$$
\begin{gathered}
L_{0}(t, \mu)=\left\{x_{0}(t, \mu) \mid z_{0}(t, \mu)=\bar{z}_{0}(t)+\Pi_{0} z^{j} \frac{\left(t-t_{0}^{j}\right)}{\mu}, t \geq t_{0}^{j},\right. \\
\left.y_{0}(t, \mu)=\bar{y}_{0}(t), t \in I\right\}
\end{gathered}
$$

существует единственное реиение $x(t, \mu)$ краевой задачи (1) - (3), пересека-

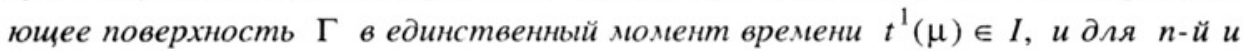
частичной суммы $X_{n}(t, \mu)$ ряда (7) справедливы оценки

$$
\begin{gathered}
\left\|y(t, \mu)-Y_{n}(t, \mu)\right\| \leq C \mu^{n+1}, \\
\left\|z(t, \mu)-Z_{n}(t, \mu)\right\| \leq C \mu^{n+1}\left[1+\alpha_{n}(t, \mu)\right], \\
\left|t_{1}(\mu)-T_{n}^{1}(\mu)\right| \leq C \mu^{n+1},
\end{gathered}
$$

где

$$
T_{n}^{1}(\mu) \equiv \sum_{l=0}^{n} \mu^{l} t_{l}^{1}, \quad \alpha_{n} \equiv \frac{1}{\mu} \exp \left(-\alpha \frac{t-\xi^{1}}{\mu}\right) n p u t \geq \xi^{1},
$$

$\alpha_{n} \equiv 0$ при $t<\xi^{1}$ для любого $\xi^{1} \in\left[T_{n}^{1}-C \mu^{n+1}, T_{n}^{1}+C \mu^{n+1}\right]$.

3. Доказательство теоремы. Обозначим через $X\left(t, \mu, x_{0}\right)$ решение задачи (1), (2) с начальным условием $X\left(0, \mu, x_{0}\right)$, через $X\left(t, 0, x_{0}\right)$ решение соответствующей вырожденной задачи (4), удовлетворяющее условию $Y(0, \mu$, $\left.x_{0}\right)=y_{0}$.

Для доказательства разрешимости краевой задачи (1) - (3) покажем, что функциональный определитель системы (3)

$$
\Lambda\left(x_{0}, \mu\right) \equiv \frac{\partial \Psi\left(x_{0}, X\left(t, \mu, x_{0}\right), \mu\right)}{\partial x_{0}}+\frac{\partial \Psi\left(x_{0}, X\left(t, \mu, x_{0}\right), \mu\right)}{\partial x_{T}} \frac{\partial X\left(T, \mu, x_{0}\right)}{\partial x_{0}}
$$

отличен от нуля в некоторой $\delta$-окрестности $S_{\delta}\left(\bar{X}_{0}^{0}\right)$ точки $\bar{X}_{0}^{0}$. 
Из теоремы [2] об оценке остаточного члена асимптотического представления разрывной сингулярно возмущенной задачи Коши вытекает, что существуют $\mu_{0}>0$ и $\delta>0$ такие, что

$$
\left\|X\left(T, \mu, x_{0}\right)-X\left(T, 0, x_{0}\right)\right\| \leq C \mu
$$

при $\mu \in\left(0, \mu_{0}\right]$ и $x_{0} \in S_{\delta}\left(\bar{X}_{0}^{0}\right)$.

Для решений систем в вариациях разрывных сингулярно возмущенных задач, которые при условиях 3 - 6 теоремы являются сингулярно возмущенными системами с асимптотически большим импульсным воздействием, следует [3], что для некоторого $\mu_{0}>0$ при $\mu \in\left(0, \mu_{0}\right]$

$$
\left\|\frac{\partial X\left(T, \mu, x_{0}\right)}{\partial x_{0}}-\frac{\partial X\left(T, 0, x_{0}\right)}{\partial x_{0}}\right\| \leq C \mu .
$$

Следовательно, из соотношений (10) - (12) заключаем, что

$$
\Lambda\left(x_{0}, \mu\right)=\Lambda_{0}\left(x_{0}\right)+O(\mu) .
$$

В силу условия 1 , представления (13) и непрерывности функции (10) по $x_{0}$ существует $\delta>0$ такое, что $\Lambda\left(x_{0}, \mu\right) \neq 0$ при $x_{0} \in S_{\delta}$.

Остальные рассуждения полностью повторяют доказательство [1] для непрерывной сингулярно возмущенной краевой задачи с одним пограничным слоем.

1. Васильева А. Б., Бутузов В. Ф. Асимптотические разложения решений сингулярно возмущенных уравнений. -- М.: Наука, 1973. -272 c.

2. Мельник T. A. Асимптотика решения некоторой разрывной сингулярно возмущенной задачи Коши // Укр. мат. журн. - 1994. - 46, № 11. - С. 1502-1508.

3. Плотников В. А. Асимптотические методы в теории дифференциальных уравнений с разрывной правой частью. - Киев, 1993. - 60 с. - (Препринт / НАН Украины. Ин-т математики; 93.27) 